\title{
Accurately quantifying the superposition state of two different Laguerre-Gaussian modes with single intensity distribution measurement
}

\author{
Ya Xiao ${ }^{1} \cdot$ Haida $\mathrm{Liu}^{1} \cdot$ Yuewei Song ${ }^{1} \cdot$ Qizhi HanZhao $^{1}$. Fengheng Che ${ }^{1}$. \\ Xuan Fan ${ }^{1}$ - Xinhong $\mathrm{Han}^{1}$. Huichao $\mathbf{Q u}^{1}$. Weichen $\mathrm{Li}^{1}$ - Wendong $\mathrm{Li}^{1}$. \\ Yongjian $\mathrm{Gu}^{1}$ (iD
}

Received: 16 July 2021 / Accepted: 18 January 2022 / Published online: 18 February 2022

(c) The Author(s) 2022

\begin{abstract}
We develop a method to quantify the superposition state of two different LaguerreGaussian modes. By analyzing the characteristics of the intensity distribution obtained in a single measurement, including the petal number, the position and value of the extremum intensity, one can quantify the angular momentum index, the radial node index and the superposition coefficient simultaneously. Experimentally, we measure a series of superposition states, whose angular momentum index ranges from -47 to 53, radial node index from 0 to 3 and superposition weight from 0.1 to 0.9 . The average trace distance and the mean fidelity of these states are lower than $0.053 \pm$ 0.001 and higher than $0.982 \pm 0.002$, respectively. Our method can further obtain the superposition coefficient compared with previous mode verification ones and can reduce the number of measurement settings compared with the traditional quantum state tomography, thus more applicable in practice.
\end{abstract}

Keywords Quantifying superposition state $\cdot$ Laguerre-Gaussian (LG) modes · Single measurement · Quantum state tomography

Y. Xiao and H. Liu: These authors contributed equally to this work

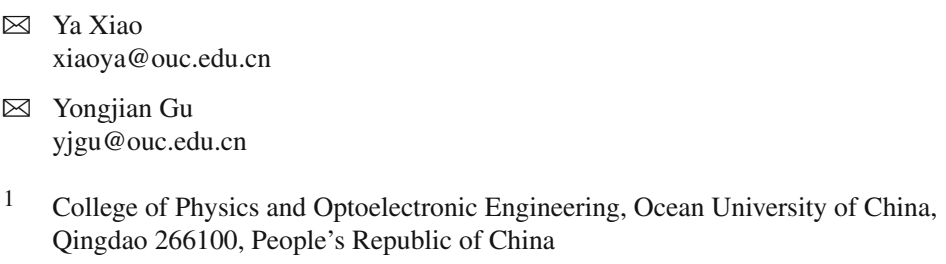




\section{Introduction}

Orbital angular momentum (OAM), a new fundamental degree of freedom of photons, has attracted increasing attention since it was discovered [1]. Its orthogonal eigenstate is theoretically infinite, and each eigenstate can be employed to encode different information. Thus, high-dimensional OAMs, especially their superposition states, can greatly improve the capacity and speed of optical communication [2-4], enhance the security of quantum information processing [5-8] and realize super-resolution imaging [9-11] as well as high-precision measurement [12-14]. Vortex beams carrying OAMs, which have special spiral phase structure and hollow intensity distribution, can be also used for particle manipulation [15-19]. As the most typical representative of vortex beam, Laguerre-Gaussian (LG) beam is characterized both by the angular momentum index $l$ and the radial node index $p$, which can further increase the dimension. In addition, LG beam is robust against environmental disturbance and easy to prepare, making it more practical and widely used in laboratories.

To realize the applications of the LG beam mentioned above, one of the crucial issues is how to accurately quantify the parameters of the superposition state encoded in the vortex beam. These parameters include angular momentum index, radial node index, superposition coefficient and relative phase. Numerous methods have been developed to detect the mode indices of vortex beams, such as interference [20,21], diffraction $[22,23]$, mapping OAM into transverse momentum [24, 25] and weak measurement [26]. However, these methods cannot simultaneously quantify all the parameters of the superposition state.

In principle, one can obtain all the parameters of the superposition state of LG beams with quantum state tomography (QST). It is a powerful method that can reconstruct the full quantum state from suitable measurements, which is mainly adopted in the $n$ qubits and $n$ qutrits systems $[27,28]$. Nevertheless, scaling QST to high-dimensional LG beam system is challenging due to the following reasons. First, with the increase in the dimension $d$, the number of measurement settings required by QST grows as $d^{2 n-1}$, where $n$ is the size of quantum system. Second, it is not clear how to find the appropriate measurements in a large $d$-dimensional system to reconstruct its state. Third, during the state tomography procedure, one needs to convert the LG mode into the fundamental mode of the single-mode fiber efficiently. This is difficult to achieve in the long distance communication process because of environmental disturbance. Thus, it is necessary to find an efficient and reliable method to fully quantify the high-dimensional superposition state of LG beams.

In this paper, we propose a method to directly quantify the superposition states of two different LG modes. Based on the analysis of the characteristics of the intensity distribution, including the petal number, the position and the value of the extremum intensity, we establish a theoretical relationship between the above characteristics and the state parameters we want to obtain then demonstrate it experimentally. Our paper is organized as follows. In Sect. 2, we present the theoretical analysis and concrete steps used to quantify the superposition state. It is divided into two situations: in one case, the absolute values of angular momentum indices are different and the radial node indices are equal to zero; in another case, the absolute values of angular momentum indices are equal and the radial node indices are unequal. In Sect. 3, we demonstrate 
the practicability of our method. The average trace distance and mean fidelity of the quantified quantum states are lower than $0.053 \pm 0.001$ and higher than $0.982 \pm 0.002$, respectively. In Sect. 4, we discuss our results and point out future directions.

\section{Theoretical model and methods}

The superposition state of two different LG modes with the same waist radius can be represented as

$$
\begin{aligned}
& \left|\psi_{s p}\right\rangle=\sin \theta\left|l_{1}, p_{1}\right\rangle+\cos \theta e^{i \phi}\left|l_{2}, p_{2}\right\rangle, \\
& \theta \in[0, \pi / 2], \quad \phi \in[0,2 \pi],
\end{aligned}
$$

where $|l, p\rangle=\sqrt{\frac{2 p !}{\pi(p+|l|) !}} \frac{1}{\omega_{z}}\left(\frac{\sqrt{2} r}{\omega_{z}}\right)^{|l|} \exp \left(\frac{-r^{2}}{\omega_{z}^{2}}\right) L_{p}^{|l|}\left(\frac{2 r^{2}}{\omega_{z}^{2}}\right) \exp \left(-\frac{i k r^{2} z}{2\left(z^{2}+z_{R}^{2}\right)}-\right.$ $\left.i l \varphi-i \xi_{z}\right)$ is the normalized complex amplitude of a single LG mode [1] with angular momentum index $l$ and radial node index $p, \omega_{z}=\omega_{0} \sqrt{1+z^{2} / z_{R}^{2}}$ is the radius of the beam at an arbitrary propagation distance $z, k=2 \pi / \lambda$ is the wave vector, $z_{R}=\pi \omega_{0}^{2} / \lambda$ is the Rayleigh range, $\xi_{z}=(2 p+|l|+1) \arctan \left(z / z_{R}\right)$ is the Gouy phase. And the intensity distribution of the superposition state can be expressed as

$$
I=\sin ^{2} \theta I_{1}+\cos ^{2} \theta I_{2}+\sin 2 \theta \operatorname{Re}\left(e^{i \phi}\left\langle l_{1}, p_{1} \mid l_{2}, p_{2}\right\rangle\right)
$$

where $I_{1}=\left\langle l_{1}, p_{1} \mid l_{1}, p_{1}\right\rangle, I_{2}=\left\langle l_{2}, p_{2} \mid l_{2}, p_{2}\right\rangle,\left\langle l_{1}, p_{1} \mid l_{2}, p_{2}\right\rangle=\sqrt{I_{1} I_{2}} \exp \left(i\left(l_{1}-\right.\right.$ $\left.\left.l_{2}\right) \varphi+i \triangle \xi_{z}\right), \triangle \xi_{z}=\left(2 p_{1}-2 p_{2}+\left|l_{1}\right|-\left|l_{2}\right|\right) \arctan \left(z / z_{R}\right)$, and $\operatorname{Re}(u)$ indicates the real part of $u$. Obviously, the intensity distribution of the superposition state is petal shaped, which arises from the $\operatorname{Re}\left(e^{i \phi}\left\langle l_{1}, p_{1} \mid l_{2}, p_{2}\right\rangle\right)$ term. The azimuthal position of the extremum intensity is located at $\varphi_{n}=\left(n \pi-\triangle \xi_{z}-\phi\right) /\left(l_{1}-l_{2}\right), n=0,1 \ldots 2 \mid l_{1}-$ $l_{2} \mid-1$. And the information of the angular momentum index, the radial node index, the superposition coefficient, as well as the relative phase we want to obtain is included in the petal number, the position and value of the extremum intensity. Thus, we can fully quantify the superposition state of two different LG modes presented in Eq. (1) by analyzing these main characteristics. For simplicity, we concentrate on the case of $\phi=0$. The steps to quantify the superposition state are divided into the following two cases.

Case $1\left|l_{1}\right| \neq\left|l_{2}\right|$.

First, we can obtain the possible combinations of $\left(l_{1}, l_{2}\right)$ according to the observed number of interference petals $N$ located on the same circle. With the azimuthal position of the extremum intensity, it is easy to demonstrate $N=\left|l_{1}-l_{2}\right|$. When $l_{1} l_{2}<0$, the number of the possible combinations of $\left(l_{1}, l_{2}\right)$ is equal to $N-1$ if $N$ is odd, otherwise it is equal to $N-2$. The minimum angular momentum index $\min \left(\left|l_{1}\right|,\left|l_{2}\right|\right)$ lies in $[1, \operatorname{Round}(N / 2)]$, i.e., $\min \left(\left|l_{1}\right|,\left|l_{2}\right|\right) \in[1, \operatorname{Round}(N / 2)]$, where $\operatorname{Round}(u)$ means rounding $u$ down to the nearest integer. On the other hand, if $l_{1} l_{2}>0$, the number of the possible combinations of $\left(l_{1}, l_{2}\right)$ is infinite; thus, we need to limit it with 
the radial position of the maximum intensity $r_{m}$. Since $r_{m} \in\left[\omega_{z} \sqrt{\left|l_{1}\right| / 2}, \omega_{z} \sqrt{\left|l_{2}\right| / 2}\right]$, $2 r_{m}^{2} / \omega_{z}^{2}-N<\min \left(\left|l_{1}\right|,\left|l_{2}\right|\right)<2 r_{m}^{2} / \omega_{z}^{2}$, see Appendix A for more details. Thus, combining the azimuthal and radial position of the maximum intensity, we can obtain the number of the possible combinations of $\left(l_{1}, l_{2}\right)$, which is equal to $3 N-1$ if $N$ is odd; otherwise, it equals to $3 N-2$.

Second, in order to find the closest combination of $\left(l_{1}, l_{2}\right)$ to the actual prepared value from above $3 N-1$ (or $3 N-2$ ) possible combinations, we define an evaluation function $\gamma$ with the experimental extremum intensity $\left(I_{\max }^{e}, I_{\min }^{e}\right)$ and the theoretical intensity $\left(I_{1}, I_{2}\right)$ of the selected combination of $\left(l_{1}, l_{2}\right)$, which is shown as follows

$$
\gamma=\left|\left(\frac{\sqrt{I_{\text {max }}^{e}}+\sqrt{I_{\text {min }}^{e}}}{2 \sqrt{I_{1}}}\right)^{2}+\left(\frac{\sqrt{I_{\text {max }}^{e}}-\sqrt{I_{\text {min }}^{e}}}{2 \sqrt{I_{2}}}\right)^{2}-1\right|
$$

Theoretically, $\gamma=0$ when the selected combination is consistent with the actual one, see Appendix B for more details.

Third, due to the symmetry of the intensity distribution, for arbitrary $l$, the intensity distribution of $|l, 0\rangle$ equals to that of $|-l, 0\rangle$. Thus, the $\gamma$ 's values of $\left(l_{1}, l_{2}\right)$ and $\left(-l_{1},-l_{2}\right)$ are the same and only the value of $\left(\left|l_{1}\right|,\left|l_{2}\right|\right)$ can be obtained by Eq. (3). However, noting that the azimuthal position of the maximum intensity of $\left(l_{1}, l_{2}\right)$ and $\left(-l_{1},-l_{2}\right)$ is symmetrical with respect to the $x$ axis, the mode of superposition state can be further determined uniquely, since only one combination of $\left(l_{1}, l_{2}\right)$ coincides with the observed extremum position.

Finally, combining the determined $\left(l_{1}, l_{2}\right)$ from above steps with the corresponding theoretical extremum intensities $I_{\max }^{e}=\left(\sin \theta \sqrt{I_{1}}+\cos \theta \sqrt{I_{2}}\right)^{2}$ and $I_{\text {min }}^{e}=$ $\left(\sin \theta \sqrt{I_{1}}-\cos \theta \sqrt{I_{2}}\right)^{2}$, we can also obtain the parameter of the superposition coefficient $\theta$ through the following formula

$$
\theta^{e}=\arcsin \frac{\sqrt{\alpha}(1+\sqrt{\beta})}{\sqrt{\alpha(1+\sqrt{\beta})^{2}+(1-\sqrt{\beta})^{2}}}
$$

where $\alpha=I_{2} / I_{1}, \beta=I_{\min }^{e} / I_{\max }^{e}$. Thus, we can fully quantify the superposition state with the above four steps, which are shown in Fig. 1.

Case $2\left|l_{1}\right|=\left|l_{2}\right|$

In this case, $l_{1}, l_{2}$ can be quantified directly by the position of maximum intensity with $\omega_{z} \sqrt{\left|l_{1}\right|}$ if $l_{1}=l_{2}$, or by the number of petals with $N=\left|l_{2}-l_{1}\right|$ if $l_{1} \neq l_{2}$. But it is impossible to obtain the superposition parameter $\theta$ when $p_{1}=p_{2}$ since the intensity distribution of $\left(l_{1},-l_{2}\right)$ and $\left(-l_{1}, l_{2}\right)$ is the same. On the other hand, if $p_{1} \neq p_{2}$, the azimuthal positions of extremum intensity are symmetrical about $x$ axis, see Appendix $\mathrm{C}$ for more details. The maximum number of the possible combinations of $\left(p_{1}, p_{2}\right)$ can be obtained by the number of the interference ring along the radial direction $M$, i.e., $\max \left(p_{1}, p_{2}\right)=M-1$. When $p_{1}>p_{2}, p_{1}=M-1, p_{2} \in[0, M-1)$; otherwise $p_{2}=M-1, p_{1} \in[0, M-1)$. Thus, the maximum number of the possible combinations of $\left(l_{1}, p_{1}\right)$ and $\left(l_{2}, p_{2}\right)$ is $2 M-2$ when $\left|l_{1}\right|=\left|l_{2}\right|$ and $p_{1} \neq p_{2}$. 


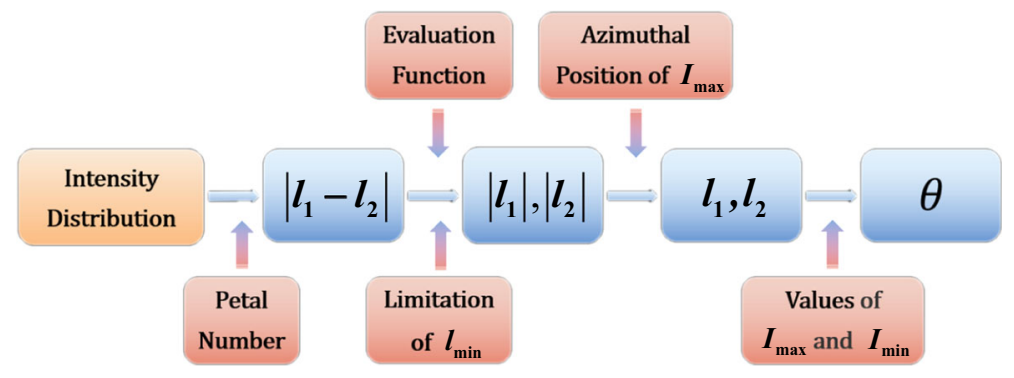

Fig. 1 Method to quantify the superposition state of two different LG modes with a single intensity distribution measurement. The light red boxes represent the characteristics of the intensity distribution used in each step. The blue boxes represent the obtained information after each step (Color figure online)

Then, with the steps similar to case 1, the superposition state shown in Eq. (1) can be obtained.

To quantify the accuracy of our method, we introduce the assemblage trace distance $D$ and fidelity $F$ between the experimentally quantified state $\rho^{e}$ and the theoretical state $\rho^{t}$, which are defined as [29]

$$
D=\frac{1}{2} \operatorname{Tr}\left(\left|\rho^{e}-\rho^{t}\right|\right), \quad F=\left(\operatorname{Tr} \sqrt{\sqrt{\rho^{e}} \rho^{t} \sqrt{\rho^{e}}}\right)^{2} .
$$

\section{Experimental setup and results}

Figure 2 schematically depicts the experimental setup to generate and quantify the superposition state of LG beam. A TEM 00 mode 405-nm laser (Toptica DLC pro) is focused on a $15 \mathrm{~mm}$ long type-II periodically poled KTiPO4 (PPKTP) crystal through a lens $\mathrm{f} 1=75 \mathrm{~mm}$ to produce spontaneous parametric down-conversion (SPDC) photons at the central wavelength of $810 \mathrm{~nm}$. The down-converted photons are collimated with another lens $\mathrm{f} 2=75 \mathrm{~mm}$ and filtered by an interference filter (IF) centered at $810 \mathrm{~nm}$ with a bandwidth of $3 \mathrm{~nm}$ (LL01-810, Semrock). In our experiment, the polarization of the pump light is set to the horizontal direction and the power to $2 \mathrm{~mW}$. The corresponding brightness of the SPDC photons reaches 100, 000 pairs/s. After separating them with a polarization beam splitter (PBS), the idler photon is directly detected by a singlephoton avalanche detector (SPAD). The detected electronic signal is used as a trigger for the camera that images the signal photon. To make sure the detection is made in time, the signal photon is delayed by a $85 \mathrm{~m}$ long single-mode fiber. After passing through lenses $\mathrm{f} 3=50 \mathrm{~mm}$ and $\mathrm{f} 4=100 \mathrm{~mm}$, it is expanded to $3 \mathrm{~mm}$ and collimated to a reflective phase-only SLM (X13138, Hamamatsu) with $1272 \times 1024$ pixels $(12.5$ $\mu \mathrm{m}$ pixel pitch) to generate any states given in Eq. (1). A pair of half-wave plates before the fiber couplers is used to modify the polarization to optimize the diffraction efficiency of SLM. The intensity distribution of the generated states is captured by a time-resolved enhanced camera (TRC411-S-GS-F) of $1600 \times 1088$ pixels $(9 \mu \mathrm{m}$ pixel 


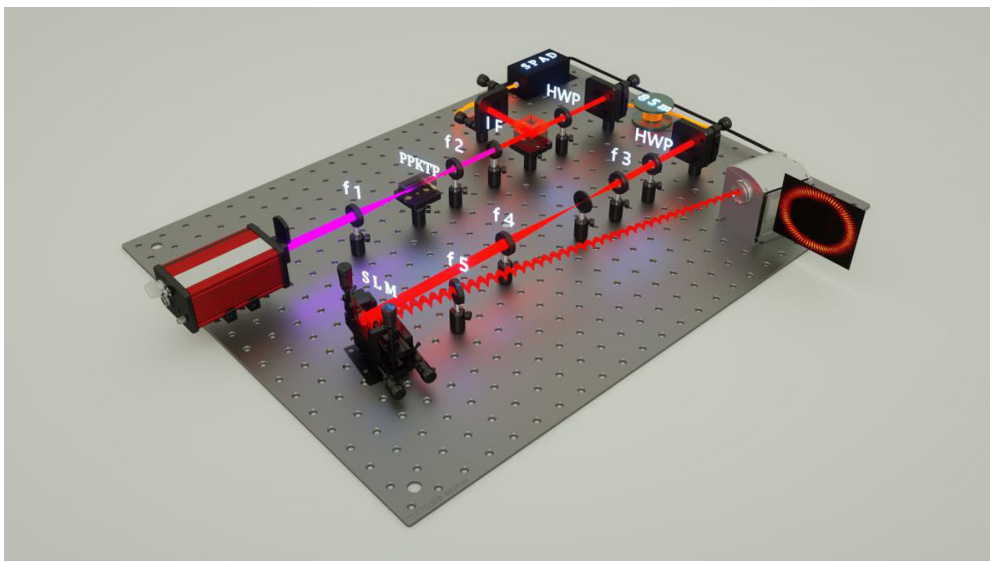

Fig. 2 Experimental setup. A TEM 00 mode 405-nm laser (Toptica DLC pro) is focused on a 15-mmlong type-II PPKTP crystal to generate the SPDC photons. The polarization of the pump light is set to the horizontal direction and the power to $2 \mathrm{~mW}$. The SPDC photons are filtered by an interference filter centered at $810 \mathrm{~nm}$ with a bandwidth of $3 \mathrm{~nm}$ (LL01-810, Semrock). The brightness is about 100, 000 pairs/s when the PPKTP crystal is operated at the degenerate temperature of $31.00 \pm 0.01^{\circ} \mathrm{C}$. After separating the SPDC photons with a polarization beam splitter, the idler photon is directly detected by a single-photon avalanche detector. The detected electronic signal is used as a trigger for the camera that images the signal photon. A 85-m-long single-mode fiber is utilized to delay the signal photon to meet the detecting time in the time-resolved enhanced camera. The lenses systems $\mathrm{f} 3$ and $\mathrm{f} 4$ expand the beam to make full use of the area of SLM to generate different perfect supposition states of LG beam. As SLM is polarization sensitive, the half-wave plates located before the fiber couplers optimize the diffraction efficiency. The intensity distribution of the generated LG beam is detected by a time-resolved enhanced camera

pitch) which is located at the focal plane of lens $f 5=500 \mathrm{~mm}$. The captured intensity image is used to quantify the prepared superposition state.

Here, we take $(|-3,0\rangle+|4,0\rangle) / \sqrt{2}$ as an example to present the concrete steps to quantify the prepared state with the experimentally observed intensity distribution, as shown in Fig. 3a. First, we binarize the intensity distribution by employing the maximum inter class variance method [30] to get the petal number. To more accurately determine the position and value of the extremum intensity, we take each pixel as the center and divide the intensity distribution into many subregions with a size of $5 \times 5$ pixels. For a given petal, the position of maximum intensity is considered to be the center of its maximum average intensity subregion, which is marked as a red square in Fig. 3a. Theoretically, these squares are on the same circle. Using the least square method to minimize the algebraic distance, we get a fitting circle with radius of $r=49.5$ pixels. The relative residual of the fitting circle is 0.073 . The maximum and minimum intensities $I_{\max }^{e}$ and $I_{\min }^{e}$ can also be determined from multipeak fitting analysis, see Fig. 3b. With these extremum intensities and Eq. (3), we then can calculate the values of evaluation function of all possible combinations of $\left(\left|l_{1}\right|,\left|l_{2}\right|\right)$, which are shown in Fig. 3c. Obviously, the smallest difference between experimental and theoretical intensity distribution is achieved at $\left|l_{1}\right|=3$ and $\left|l_{2}\right|=4$. It means the combination of $\left(l_{1}, l_{2}\right)$ of the prepared state is $(-3,4)$ or $(3,-4)$. By comparing the azimuthal position of the experimental extremum intensity with that of the theoretical extremum intensity, we can further obtain $l_{1}=-3$ and $l_{2}=4$. Submitting them into 
(a)
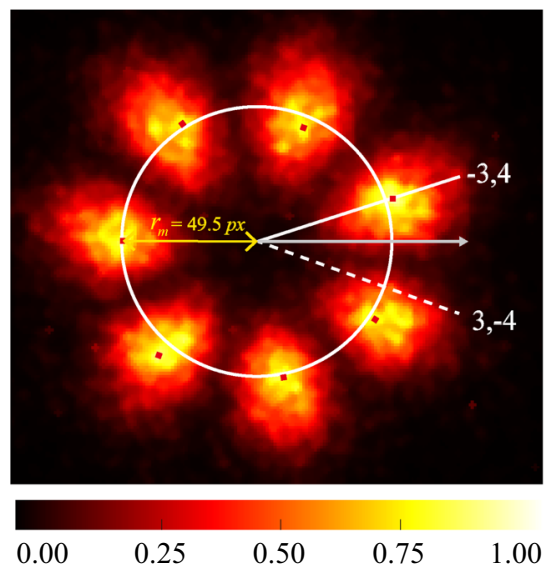

Distribution of normalized intensity (c)
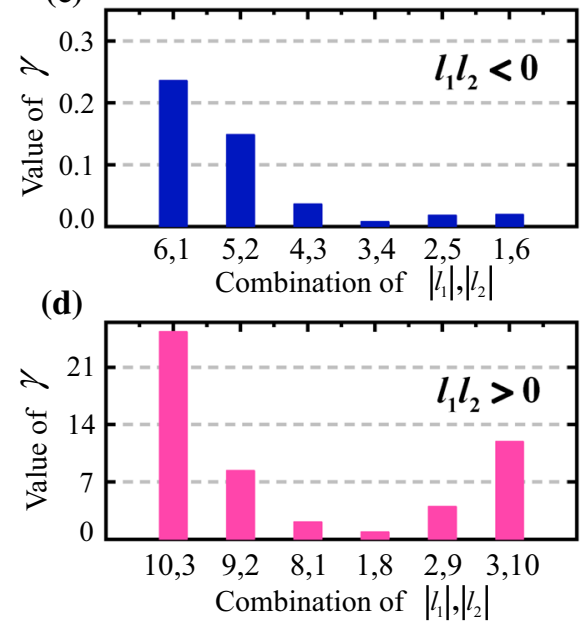

(b)

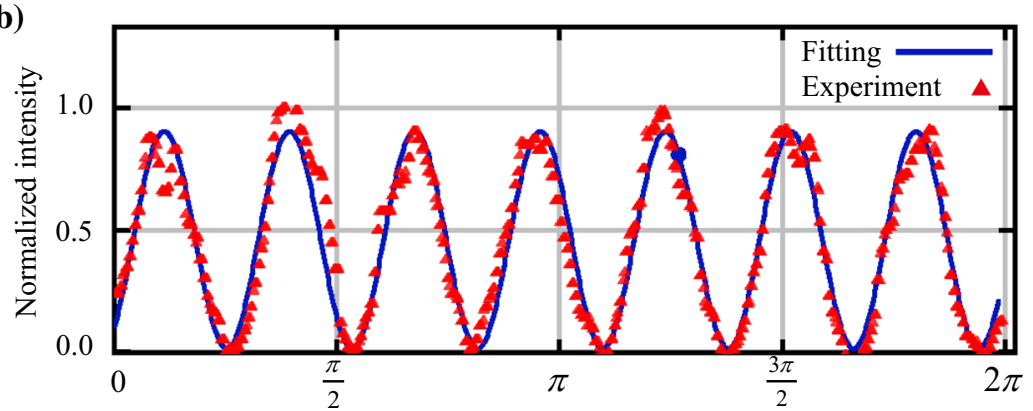

Fig. 3 Experimental results obtained after each quantification step for the prepared supposition state $(\mid-$ $3,0\rangle+|4,0\rangle) / \sqrt{2}$. a The experimentally observed normalized intensity distribution. The white circle represents the fitted position of the maximum intensity whose radius is equal to 49.5 pixels. $\mathbf{b}$ The relationship between the normalized intensity and the azimuthal angle on the fitted circle. $\mathbf{c}$ and $\mathbf{d}$ The corresponding values of evaluation function $\gamma$ under different combinations of $\left|l_{1}\right|,\left|l_{2}\right|$. Error bars are estimated by the Poissonian statistics of two-photon coincidences, which are about 0.001 in our experiment

Eq. (4), we finally get $\theta^{e}=0.784$, which is very close to the theoretical value $\pi / 4$. The fidelity of our quantified state is $0.991 \pm 0.007$.

We further analyze the second minimum value of the evaluation function and the Gouy phase corresponding to the maximum intensity with 48 different superposition states with $l_{1}$ spanning from -3 to $3, l_{2}$ from -5 to 5 when $p_{1}=p_{2}=0$ and $\theta=\pi / 4$. Figure $4 \mathrm{a}$ and $\mathrm{d}$ presents the minimum value of the evaluation function $\gamma$ and the ratio of its minimum value to the sub minimum value $\kappa$. The smaller the value is, the more accurately the angular momentum indices combination can be obtained. The ratio of the Gouy phases of high-order mode to the zero-order mode $\eta=\Delta \xi_{z} / \arctan \left(\frac{z}{z_{R}}\right)$ is shown in Fig. 4b and e. Obviously, the experimental results agree well with the theory, which further confirms the feasibility of our method. Figure $4 \mathrm{c}$ and $\mathrm{f}$ presents the trace distances between the quantified states and their corresponding theoretical states, 

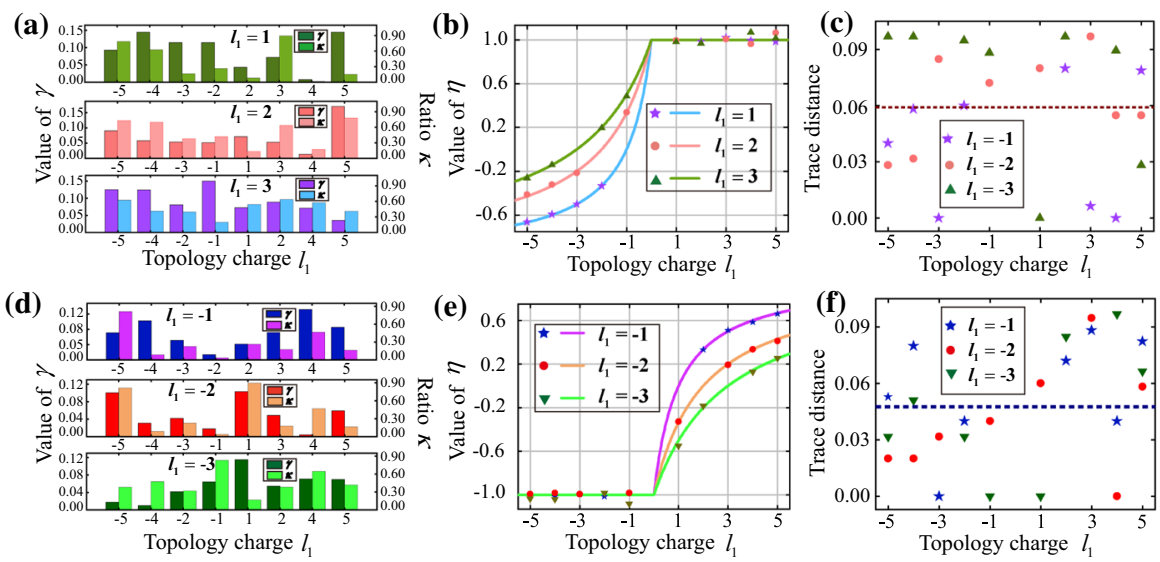

Fig. 4 Experimental results of different combinations of $\left(l_{1}, l_{2}\right)$ with $\theta=\pi / 4$. a, $\mathbf{d}$ The minimum value of the evaluation function $\gamma$ and the ratio of its minimum value to the sub minimum value $\kappa$. $\mathbf{b}, \mathbf{e}$ The ratio of the Gouy phases of high-order superposition mode to the zero-order superposition mode $\eta=\Delta \xi_{z} / \arctan \left(\frac{z}{z_{R}}\right)$. c, $\mathbf{f}$ The trace distances between the quantified states and their corresponding theoretical states. The dashed lines represent the corresponding average values. Error bars are estimated by the Poissonian statistics of two-photon coincidences, which are about 0.001

whose average values are $0.059 \pm 0.001$ and $0.047 \pm 0.001$, respectively, indicating a high accuracy of the quantified state.

In order to demonstrate the universality of our method, we increased the superposition weight $\sin ^{2} \theta$ from 0.1 to 0.9 in steps of 0.2. As shown in Fig. $5 a$ and $b$, the average fidelity of these states is $0.987 \pm 0.004$. In addition, we also test a series of states with large angular momentum index and non-zero radial node index. The experimental results are shown in Fig. 5c and d, respectively. Due to the influence of environmental noise, the interference visibility will decrease with the increase in the mode indices. However, larger indices provide more petals, which can give more information about the extreme intensity; thus, the average fidelity of these states quantified by our method is still remaining above $0.982 \pm 0.002$.

\section{Discussion and conclusion}

In this work, we theoretically develop and experimentally demonstrate that it is feasible to directly quantify the superposition state of two different LG modes by a single intensity distribution measurement. By analyzing the characteristics of the intensity distribution, including the petal number, the position and the value of the extremum intensity, we present the concrete steps which quantify the superposition state. Experimentally, we measure a series of superposition states with the angular momentum index ranging from -47 to 53 , the radial node index from 0 to 3 and the superposition weight from 0.1 to 0.9 . The average trace distance of these states is lower than $0.053 \pm 0.001$ and their mean fidelity is higher than $0.982 \pm 0.002$, demonstrating the reliability of our method in different high-dimension space. 

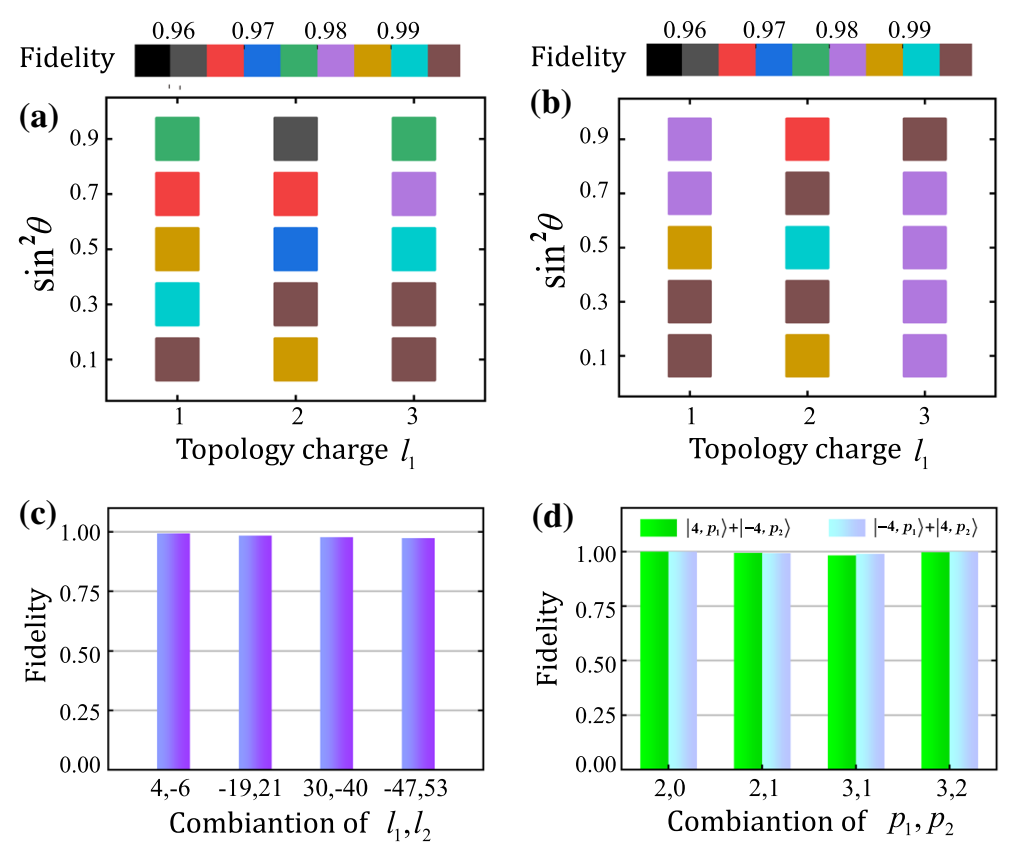

Fig. 5 a Fidelities of different superposition states with four petals and $l_{1} l_{2}>0$ (a), with seven petals and $l_{1} l_{2}<0(\mathbf{b})$, with large angular momentum index $(\mathbf{c})$, as well as non-zero radial node index $\mathbf{d}$ All the fidelities of these states are above 0.974. Error bars are estimated by the Poissonian statistics of two-photon coincidences, which are about 0.001

Compared with the previous mode quantification methods, our method can further quantify the superposition weight of various modes present in the vortex beam, which has potential application in mode demultiplexing [31]. In addition, our method just needs to record the intensity distribution, which can mitigate the difficulties appearing in the traditional QST, such as finding the appropriate measurement operator, conducting exponential growth measurements in high dimension as well as converting the LG mode into the fundamental mode of the single-mode fiber. In summary, our method is more practicable.

What's more, with the help of machine learning, our method can be extended to more general scenarios involving more modes or mixed states. We will carry out these researches in the near future.

Acknowledgements This work was supported by the National Natural Science Foundation Regional Innovation and Development Joint Fund (Grant No. 932021070), the National Natural Science Foundation of China (Grants No. 912122020 and No. 61701464), the China Postdoctoral Science Foundation (Grant No. 861905020051), the Fundamental Research Funds for the Central Universities (Grants No. 841912027, and No. 842041012), the Applied Research Project of Postdoctoral Fellows in Qingdao (Grant No. 861905040045), and the Young Talents Project at Ocean University of China (Grant No. 861901013107).

Data and materials availability All data generated or analyzed during this study are included in this published article (and its supplementary information files). Additional data related to this paper may be requested from the authors. 
Open Access This article is licensed under a Creative Commons Attribution 4.0 International License, which permits use, sharing, adaptation, distribution and reproduction in any medium or format, as long as you give appropriate credit to the original author(s) and the source, provide a link to the Creative Commons licence, and indicate if changes were made. The images or other third party material in this article are included in the article's Creative Commons licence, unless indicated otherwise in a credit line to the material. If material is not included in the article's Creative Commons licence and your intended use is not permitted by statutory regulation or exceeds the permitted use, you will need to obtain permission directly from the copyright holder. To view a copy of this licence, visit http://creativecommons.org/licenses/by/4.0/.

\section{References}

1. Allen, L., Beijersbergen, M.W., Spreeuw, R.J.C., Woerdman, J.P.: Orbital angular momentum of light and the transformation of Laguerre-Gaussian laser modes. Phys. Rev. A 45, 8185-8189 (1992). https:// doi.org/10.1103/PhysRevA.45.8185

2. Wang, J., Yang, J.Y., Fazal, I.M., Ahmed, N., Yan, Y., Huang, H., Ren, Y.X., Yue, Y., Dolinar, S., Tur, M., Willner, A.E.: Terabit free-space data transmission employing orbital angular momentum multiplexing. Nat. Photonics 6, 488-496 (2012). https://doi.org/10.1038/nphoton.2012.138

3. Bozinovic, N., Yue, Y., Ren, Y., Tur, M., Kristensen, P., Huang, H., Willner, A.E., Ramachandran, S.: Terabit-scale orbital angular momentum mode division multiplexing in fibers. Science 340, 1545-1548 (2013). https://doi.org/10.1126/science.1237861

4. Yan, Y., Xie, G.D., Lavery, M.P.J., Huang, H., Ahmed, N., Bao, C.J., Ren, Y.X., Cao, Y.W., Li, L., Zhao, Z., Molisch, A.F., Tur, M., Padgett, M.J., Willner, A.E.: High-capacity millimetre-wave communications with orbital angular momentum multiplexing. Nat. Commun. 5, 4876 (2014). https:// doi.org/10.1038/ncomms5876

5. Vallone, G., D’Ambrosio, V., Sponselli, A., Slussarenko, S., Marrucci, L., Sciarrino, F., Villoresi, P.: Free-space quantum key distribution by rotation-invariant twisted photons. Phys. Rev. Lett. 113, 060503 (2014). https://doi.org/10.1103/PhysRevLett.113.060503

6. Wang, X.L., Cai, X.D., Su, Z.E., Chen, M.C., Wu, D., Li, L., Liu, N.L., Lu, C.Y., Pan, J.W.: Quantum teleportation of multiple degrees of freedom of a single photon. Nature 518, 516-519 (2015). https:// doi.org/10.1038/nature14246

7. Sit, A., Bouchard, F., Fickler, R., Gagnon-Bischoff, J., Larocque, H., Heshami, K., Elser, D., Peuntinger, C., Günthner, K., Heim, B., Marquardt, C., Leuchs, G., Boyd, R.W., Karimi, E.: High-dimensional intracity quantum cryptography with structured photons. Optica 4, 1006-1010 (2017). https://doi.org/ 10.1364/OPTICA.4.001006

8. Xie, Z.W., Gao, S.C., Lei, T., Feng, S.F., Zhang, Y., Li, F., Zhang, J.B., Li, Z.H., Yuan, X.C.: Integrated (de)multiplexer for orbital angular momentum fiber communication. Photonics Res. 6, 743-749 (2018). https://doi.org/10.1364/PRJ.6.000743

9. Willig, K.I., Rizzoli, S.O., Westphal, V., Jahn, R., Hell, S.W.: STED microscopy reveals that synaptotagmin remains clustered after synaptic vesicle exocytosis. Nature 440, 935-939 (2006). https://doi. org/10.1038/nature04592

10. Xie, X.S., Chen, Y.Z., Yang, K., Zhou, J.Y.: Harnessing the point-spread function for high-resolution farfield optical microscopy. Phys. Rev. Lett. 113, 263901 (2014). https://doi.org/10.1103/PhysRevLett. 113.263901

11. Kozawa, Y., Matsunaga, D., Sato, S.: Superresolution imaging via super-oscillation focusing of a radially polarized beam. Optica 5, 86-92 (2018). https://doi.org/10.1364/OPTICA.5.000086

12. Lavery, M.P.J., Speirits, F.C., Barnett, S.M., Padgett Miles, J.: Detection of a spinning object using light's orbital angular momentum. Science 341, 537-540 (2013). https://doi.org/10.1126/science. 1239936

13. Kravets, V.G., Schedin, F., Jalil, R., Britnell, L., Gorbachev, R.V., Ansell, D., Thackray, B., Novoselov, K.S., Geim, A.K., Kabashin, A.V., Grigorenko, A.N.: Singular phase nano-optics in plasmonic metamaterials for label-free single-molecule detection. Nat. Mater. 12, 304-309 (2013). https://doi.org/10. 1038/nmat3537

14. Li, Y., Yu, L., Zhang, Y.X.: Influence of anisotropic turbulence on the orbital angular momentum modes of Hermite-Gaussian vortex beam in the ocean. Opt. Express 25, 12203-12215 (2017). https://doi.org/ 10.1364/OE.25.012203 
15. Paterson, L., MacDonald, M.P., Arlt, J., Sibbett, W., Bryant, P.E., Dholakia, K.: Controlled rotation of optically trapped microscopic particles. Science 292, 912-914 (2001). https://doi.org/10.1126/science. 1058591

16. Grier, D.G.: A revolution in optical manipulation. Nature 424, 810-816 (2003). https://doi.org/10. 1038/nature01935

17. Padgett, M., Bowman, R.: Tweezers with a twist. Nat. Photonics 5, 343-348 (2011). https://doi.org/ 10.1038/nphoton.2011.81

18. Gong, L.P., Gu, B., Rui, G.H., Cui, Y.P., Zhu, Z.Q., Zhan, Q.W.: Optical forces of focused femtosecond laser pulses on nonlinear optical Rayleigh particles. Photonics Res. 6, 138-143 (2018). https://doi.org/ 10.1364/PRJ.6.000138

19. Zhang, Y., Shen, J., Min, C., Jin, Y., Jiang, Y., Liu, J., Zhu, S., Sheng, Y., Zayats, A.V., Yuan, X.: Nonlinearity-induced multiplexed optical trapping and manipulation with femtosecond vector beams. Nano Lett. 18, 5538-5543 (2018). https://doi.org/10.1021/acs.nanolett.8b01929

20. Zhang, W., Qi, Q., Zhou, J., Chen, L.: Mimicking faraday rotation to sort the orbital angular momentum of light. Phys. Rev. Lett. 112, 153601 (2014). https://doi.org/10.1103/PhysRevLett.112.153601

21. Zhao, Q., Dong, M., Bai, Y.H., Yang, Y.J.: Measuring high orbital angular momentum of vortex beams with an improved multipoint interferometer. Photonics Res. 8, 745-749 (2020). https://doi.org/10. 1364/PRJ.384925

22. Dai, K., Gao, C., Zhong, L., Na, Q., Wang, Q.: Measuring OAM states of light beams with graduallychanging-period gratings. Opt. Lett. 40, 562 (2015). https://doi.org/10.1364/OL.40.000562

23. Hickmann, J.M., Fonseca, E.J.S., Soares, W.C., Chávez-Cerda, S.: Unveiling a truncated optical lattice associated with a triangular aperture using light's orbital angular momentum. Phys. Rev. Lett. 105, 053904053904 (2010). https://doi.org/10.1103/PhysRevLett.105.053904

24. Berkhout, G.C.G., Lavery, M.P.J., Courtial, J., Beijersbergen, M.W., Padgett, M.J.: Efficient sorting of orbital angular momentum states of light. Phys. Rev. Lett. 105, 153601 (2010). https://doi.org/10. 1103/PhysRevLett.105.153601

25. Mirhosseini, M., Malik, M., Shi, Z., Boyd, R.W.: Efficient separation of the orbital angular momentum eigenstates of light. Nat. Commun. 4, 2781 (2013). https://doi.org/10.1038/ncomms3781

26. Zhu, J., Zhang, P., Wang, F., Wang, Y., Li, Q., Liu, R., Wang, J., Gao, H., Li, F.: Experimentally measuring the mode indices of Laguerre-Gaussian beams by weak measurement. Opt. Express 29, 5419-5426 (2021). https://doi.org/10.1364/OE.416671

27. James, D.F.V., Kwiat, P.G., Munro, W.J., White, A.G.: Measurement of qubits. Phys. Rev. A 64, 052312 (2001). https://doi.org/10.1103/PhysRevA.64.052312

28. Thew, R.T., Nemoto, K., White, A.G., Munro, W.J.: Qudit quantum-state tomography. Phys. Rev. A 66, 012303 (2002). https://doi.org/10.1103/PhysRevA.66.012303

29. Nielsen, M.A., Chuang, I.L.: Quantum computation and quantum information. Cambridge University Press, Cambridge (2000). https://doi.org/10.1017/CBO9780511976667

30. Ostu, N.: A threshold selection method from gray-level histogram. IEEE Trans SMC-9, 62-66 (1979). https://doi.org/10.1109/TSMC.1979.4310076

31. Flamm, D., Hou, K.C., Gelszinnis, P., Schulze, C., Schröter, S., Duparré, M.: Modal characterization of fiber-to-fiber coupling processes. Opt. Lett. 38, 2128-2130 (2013). https://doi.org/10.1364/OL.38. 002128

Publisher's Note Springer Nature remains neutral with regard to jurisdictional claims in published maps and institutional affiliations. 